\title{
Troponin I in acute decompensated heart failure: insights from the ASCEND-HF study
}

\author{
G. Michael Felker ${ }^{1 *}$, Vic Hasselblad', W.H. Wilson Tang ${ }^{2}$, Adrian F. Hernandez', \\ Paul W. Armstrong ${ }^{3}$, Gregg C. Fonarow ${ }^{4}$, Adriaan A. Voors ${ }^{5}$, Marco Metra ${ }^{6}$, \\ John J.V. McMurray, Javed Butler ${ }^{8}$, Gretchen M. Heizer ${ }^{1}$, Kenneth Dickstein', \\ Barry M. Massie ${ }^{10}$, Dan Atar ${ }^{11}$, Richard W. Troughton ${ }^{12}$, Stefan D. Anker ${ }^{13}$, \\ Robert M. Califf ${ }^{14}$, Randall C. Starling ${ }^{2}$, and Christopher M. O'Connor'1
}

\begin{abstract}
${ }^{1}$ Duke Clinical Research Institute, Duke University Medical Center, Durham, NC 27705, USA; ${ }^{2}$ Cleveland Clinic Foundation, Cleveland, OH, USA; ${ }^{3}$ University of Alberta, Edmonton, Canada; ${ }^{4}$ Ahmanson-UCLA Cardiomyopathy Center, Ronald Reagan UCLA Medical Center, Los Angeles, CA, USA; ${ }^{5}$ University Medical Center Groningen, Groningen, The Netherlands; ${ }^{6}$ Institute of Cardiology, University of Brescia, Brescia, Italy; ${ }^{7}$ Western Infirmary, University of Glasgow, Glasgow, UK; ${ }^{8}$ Cardiology Division, Emory University, Atlanta, GA, USA; ${ }^{9}$ Stavenger University Hospital, University of Bergen, Bergen, Norway; ${ }^{10}$ San Francisco VA Medical Center, San Francisco, CA, USA; ${ }^{11}$ Department of Cardiology, Oslo University Hospital Ulleval, Norway, and Institute for Clinical Medicine, University of Oslo, Norway; ${ }^{12}$ University of Otago, Christchurch, New Zealand; ${ }^{13}$ Department of Cardiology, Applied Cachexia Research, Charité Universitätsmedizin, Berlin, Germany and Centre for Clinical and Basic Research, IRCCS San Raffaele, Rome, Italy; and ${ }^{14}$ Duke Translational Medicine Institute, Duke University Medical Center, Durham, NC, USA
\end{abstract}

Received 27 April 2012; revised 30 May 2012; accepted 1 June 2012

Aims

We examined the prognostic importance of cardiac troponin I (cTnl) in a cohort of patients enrolled in the ASCEND-HF study of nesiritide in acute decompensated heart failure (ADHF). Circulating troponins are a prognostic marker in patients with ADHF. Contemporary assays with greater sensitivity require reassessment of the significance of troponin elevation in HF.

Methods and results

Cardiac troponin I was measured in a core laboratory in 808 ADHF patients enrolled in the ASCEND-HF biomarkers substudy using a sensitive assay (VITROS Trop I ES, Ortho Clinical Diagnostics) with a lower limit of detection of $0.012 \mathrm{ng} / \mathrm{mL}$ and a 99th percentile upper reference limit (URL) of $0.034 \mathrm{ng} / \mathrm{mL}$. Patients with clinical evidence of acute coronary syndrome or troponin $>5 \times$ the URL were excluded. Multivariable modelling was used to assess the relationship between $\log (\mathrm{cTnl})$ and in-hospital and post-discharge outcomes. Baseline cTnl was undetectable in $22 \%$ and elevated above the 99 th percentile URL in $50 \%$ of subjects. cTnl levels did not differ based on HF aetiology. After multivariable adjustment, higher $\mathrm{CTnl}$ was associated with worsened in-hospital outcomes such as length of stay $(P=0.01)$ and worsening HF during the index hospitalization $(P=0.01)$, but was not associated with worsened post-discharge outcomes at 30 or 180 days. The relationship between $\mathrm{cTnl}$ and outcomes was generally linear and there was no evidence of a threshold effect at any particular level of cTnl.

Conclusion $\mathrm{cTnl}$ is elevated above the 99 th percentile URL in $50 \%$ of ADHF patients and predicts in-hospital outcome, but is not an independent predictor of long-term outcomes.

Keywords Troponin • Biomarkers • Heart failure

\section{Introduction}

Cardiac troponins are sensitive biomarkers of myocardial injury, and play a fundamental role in the diagnosis and risk stratification of patients with acute coronary syndromes (ACS). Data from multiple studies have demonstrated that circulating cardiac troponins are detectable in patients with heart failure (HF) even in the absence of clinically apparent myocardial ischaemia, and that cardiac troponins may be useful prognostic markers in patients with acute and chronic HF. ${ }^{1}$ Although the exact mechanisms of myocardial injury in HF are uncertain, ischaemia, haemodynamic stress, oxidative stress, inflammation, altered calcium handling, and impaired renal clearance have all been proposed as mechanisms of troponin elevation in $\mathrm{HF}^{1,2}$ 
Recent improvements in the sensitivity of troponin assays have complicated the interpretation of these biomarkers in HF. The increasing sensitivity of more contemporary assays has resulted in the detection of circulating troponin in a progressively greater proportion of HF patients, including many patients with troponin levels greater than the diagnostic myocardial infarction threshold [the 99th percentile upper reference limit (URL)]. This phenomenon has led to increasing uncertainty about the clinical interpretation of troponin data from contemporary assays, particularly in patients with acute decompensated HF (ADHF). A substantial proportion of ADHF patients have elevations of circulating troponin, ${ }^{3-5}$ and both the symptoms and the aetiology of ADHF may overlap significantly with those of ACS. Using a contemporary troponin assay, we analysed data from a pre-specified biomarker substudy of the ASCEND-HF (Acute Study of Clinical Effectiveness of Nesiritide in Decompensated Heart Failure) study in order to assess (i) the prevalence and clinical correlates of troponin elevation in ADHF; (ii) the association between troponin elevation and in-hospital and post-discharge clinical outcomes; (iii) the prognostic implications of changes in troponin levels during initial ADHF therapy; and (iv) the relationship between nesiritide treatment and troponin status.

\section{Methods}

The methods and primary results of the ASCEND-HF study (NCT00475852, clinical trials.gov) have been published previously., ${ }^{6,7}$ Briefly, ASCEND-HF was a multicentre, randomized, double-blind, placebo-controlled trial of nesiritide compared with placebo in 7141 patients hospitalized with ADHF. Co-primary endpoints were improvement in dyspnoea at 6 or $24 \mathrm{~h}$ as measured by a 7-point Likert scale and HF hospitalization or death at 30 days. Patients with clinical evidence of ACS or baseline troponin level $>5 \times$ the URL at the local clinical laboratory were excluded from the trial. A subgroup of patients from the ASCEND-HF study were enrolled in a biomarker substudy $(n=808)$. Blood samples were obtained in serum and EDTA plasma at baseline, $48-72 \mathrm{~h}$, and 30 days, and were immediately centrifuged and stored at $-80^{\circ} \mathrm{C}$ for subsequent analysis. At a central core laboratory blinded to all clinical data, cardiac troponin I (cTnl) was measured in serum samples using a contemporary 'guideline-acceptable ${ }^{, 8}$ sensitive assay (VITROS Trop I ES, Ortho Clinical Diagnostics, Raritan, $\mathrm{NJ}$, USA) with a lower limit of detection of $0.012 \mathrm{ng} / \mathrm{mL}$, a 99th percentile URL of $0.034 \mathrm{ng} / \mathrm{mL}$, and a coefficient of variation of $10 \%$ at the 99th percentile URL. N-terminal pro brain natriuretic peptide (NT-proBNP) was measured on serum samples at the same core laboratory using a clinically available assay (VITROS NTproBNP, Ortho Clinical Diagnostics).

\section{Statistical analysis}

Categorical variables were described as counts and percentages, and continuous variables were summarized as medians (25th, 75th percentiles). Differences in binary variables were calculated using the $\chi^{2}$ test. Continuous and ordinal variables were compared using the Wilcoxon rank-sum test. Because cTnl was not normally distributed, this variable was log transformed in all analyses using log base 2, such that reported hazard ratios (HRs) and odds ratios (ORs) represented the risk associated with a doubling of cTnl. All ORs and HRs are reported as point estimates with $95 \%$ confidence intervals $(\mathrm{Cls})$. For the purposes of analysis, levels below the lower limit of detection $(<0.012 \mathrm{ng} / \mathrm{mL})$ were imputed as $0.006 \mathrm{ng} / \mathrm{mL}$, since this provided the best statistical fit for the observed distribution of troponin data. We used multivariable linear regression to identify clinical predictors of elevated $\mathrm{cTnl}$ at baseline. The association between cTnl and outcomes was determined using both univariable and multivariable logistic regression analysis or Cox proportional hazards analysis (for length of stay and 180-day mortality). The clinical endpoints of interest were (i) 30-day mortality; (ii) the composite of 30-day mortality and rehospitalization for HF; (iii) 180-day mortality; (iv) dyspnoea improvement at 6 and 24 h; (v) worsening HF or death up to day 7; and (vi) length of stay for the index hospitalization. For multivariable analysis, adjusted models for each endpoint of interest ('final adjusted models') were constructed in the overall ASCEND-HF population (see Appendix for the final list of covariates for each endpoint). These covariates were then used to perform multivariable adjustment in the biomarker substudy subset reported herein. Models were tested with and without the inclusion of NT-proBNP. We also examined the interaction between randomized treatment (nesiritide or placebo) and troponin status with regard to each outcome using an interaction term (treatment $x$ cTnl) in the multivariable model for each endpoint.

To evaluate the relationship between change in cTnl during the acute hospitalization (between baseline and 48-72 h) and subsequent outcomes, we evaluated both the absolute change in troponin values and a $20 \%$ change in troponin from baseline. Both these analyses excluded patients with undetectable troponin at baseline. Finally, we examined the significance of a rise or fall in troponin that crossed the 99th percentile URL, consistent with the universal definition of myocardial infarction. ${ }^{9}$

\section{Results}

\section{Patient population}

The biomarker substudy population $(n=808)$ was broadly similar to the overall ASCEND-HF population $(n=7141)$, with the exception that $85 \%$ of patients in the biomarker substudy were enrolled in North America (compared with $43 \%$ in the main trial). The median cTnl level was $0.034 \mathrm{ng} / \mathrm{mL}(0.012,0.058)$. Baseline cTnl was below the lower limit of detection $(<0.012 \mathrm{ng} / \mathrm{mL})$ in $22 \%$ and elevated above the 99 th percentile URL in $50 \%$. The proportion of patients with cTnl levels above the 99th percentile URL decreased at each time point, but remained substantial (31\%) even at day 30 (Figure 1). Baseline characteristics for the study population stratified by $\mathrm{cTnl}$ above or below the 99th percentile URL (which was also the median cTnl level) are shown in Table 1. Patients with higher cTnl levels had other characteristics associated with higher risk, including being older, more often male, and having higher NT-proBNP and worse renal function $(P<0.05$ for all). Notably, cTnl levels did not differ based on whether patients had HF of ischaemic vs. non-ischaemic aetiology (median $\mathrm{cTnl}=$ $0.034 \mathrm{ng} / \mathrm{mL}$ for ischaemic vs. $0.035 \mathrm{ng} / \mathrm{mL}$ for non-ischaemic; $P=$ 0.77). Patients with HF and preserved ejection fraction ( $\geq 40 \%$ ) had significantly lower $c T n l$ levels (median $=0.026 \mathrm{ng} / \mathrm{mL}$ ) than those with systolic dysfunction (median $=0.037 ; P=0.03$ ) (Figure 2 ).

\section{Correlates of elevated baseline troponin}

Variables that were significantly associated with elevated cTnl at baseline are shown in Table 2. The factor with by far the strongest association with elevated cTnl was elevated NT-proBNP $(P<0.0001)$. Region was also a significant predictor of elevated cTnl, with patients enrolled in North America or Latin America 
having lower cTnl levels than those enrolled in other regions (Western Europe, Central Europe, or Asia).

\section{Association of baseline troponin and outcomes}

\section{In-hospital outcomes}

For analysis of the relationship between cTnl and dyspnoea response, dyspnoea was dichotomized as moderately or markedly improved (based on the Likert scale) at 6 and 24 hrelative to the

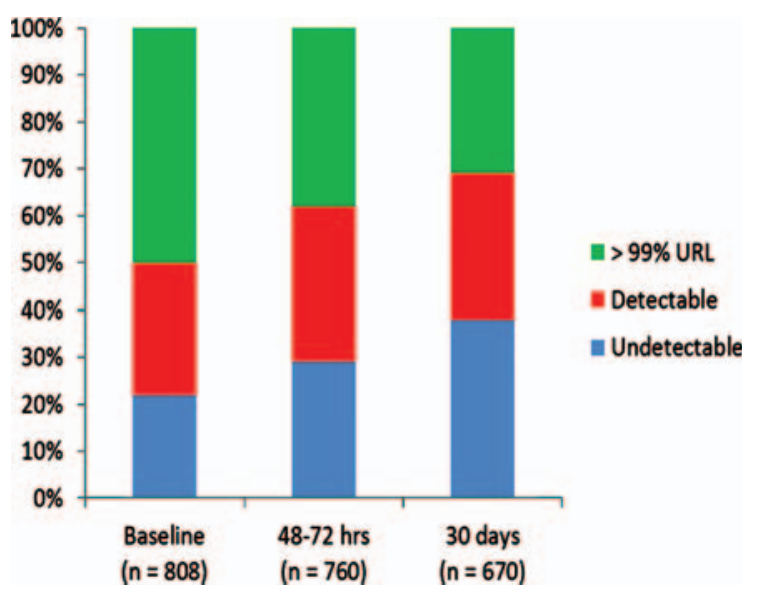

Figure I Proportion of patients at each time point with troponin I values below the lower limit of detection $(<0.012 \mathrm{ng} / \mathrm{mL})$, between the lower limit of detection and the 99th percentile URL $(\geq 0.012 \mathrm{ng} / \mathrm{mL}$ to $\leq 0.034 \mathrm{ng} / \mathrm{mL})$, and greater than the 99th percentile URL ( $>0.034 \mathrm{ng} / \mathrm{mL})$. URL, upper reference limit. time of randomization. Baseline cTnl was not a significant predictor of dyspnoea relief at $6 \mathrm{~h}$ (adjusted OR 1.04, 95\% Cl 0.92-1.18) per doubling of $\mathrm{cTnl} ; P=0.502$ ), but troponin elevation was modestly associated with lower odds of dyspnoea relief at $24 \mathrm{~h}$ (adjusted OR 0.85, 95\% Cl 0.72-0.99 per doubling of cTnl; $P=0.035$ ) (Table 3).

Baseline troponin status was a strong predictor of other clinical outcomes during the index hospitalization. Specifically, increased baseline cTnl was associated with a higher risk of the endpoint of death or worsening HF prior to discharge in both univariate (OR 1.31, 95\% Cl 1.12-1.54 per doubling of cTnl; $P=0.001$ ) and multivariable analysis (OR 1.34, 95\% Cl 1.10-1.62 per doubling of $\mathrm{cTnl} ; P=0.013$ ) (Figure $3 A$ ). Increases in baseline $\mathrm{cTnl}$ were also associated with a higher risk for increased length of stay in both univariate analysis ( $\mathrm{HR} \mathrm{0.93,95 \%} \mathrm{Cl} \mathrm{0.89-0.97;} P=$ $0.001)$ and after adjustment for other known predictors (HR $0.95,95 \% \mathrm{Cl} 0.91-0.99 ; P=0.013)$.

\section{Long-term outcomes}

Event rates were 3.1\% $(n=25)$ for 30-day mortality, 12.1\% $(n=$ 98) for the composite of death or rehospitalization at 30 days, and $11.5 \%(n=93)$ for 180-day mortality. The univariable and adjusted relationships between baseline cTnl and 30- and 180-day outcomes are shown in Table 3. Higher baseline cTnl was associated with a higher 30-day mortality rate in univariate analysis (OR 1.23, 95\% Cl 1.01-1.50 per doubling of cTnl; $P=0.035)$. However, after adjustment for other predictors, cTnl was no longer associated with 30-day mortality (adjusted OR 1.11, 95\% Cl 0.89-1.38 per doubling of cTnl; $P=0.37$ ) (Figure 3B). For the composite endpoint of death or HF rehospitalization at 30 days, elevated cTnl was not significantly associated with outcome in either univariable (OR 1.09, 95\% Cl 0.97-1.22;

Table I Baseline characteristics stratified by troponin I above or below the 99th percentile upper reference limit

\begin{tabular}{|c|c|c|c|c|}
\hline & Total $(n=808)$ & cTnl $\leq 0.034 \mathrm{ng} / \mathrm{L}(n=404)$ & cTnl $>0.034 \mathrm{ng} / \mathrm{mL}(n=404)$ & $P$-value \\
\hline Age (years) & 67 & 65 & 69 & 0.022 \\
\hline Sex (male, \%) & 70.1 & 64.6 & 75.5 & 0.0007 \\
\hline Race (White, \%) & 68.2 & 68.8 & 67.6 & 0.84 \\
\hline Systolic BP (mmHg) & 124.0 & 124.0 & 124.5 & 0.52 \\
\hline Heart rate (b.p.m.) & 79.0 & 79.0 & 79.0 & 0.82 \\
\hline Atrial fibrillation (\%) & 41.3 & 40.6 & 42.1 & 0.67 \\
\hline Hypertension (\%) & 77.7 & 80.2 & 75.3 & 0.091 \\
\hline NT-proBNP (pg/mL) & 3700 & 2980 & 4554 & 0.0008 \\
\hline Serum creatinine $(\mu \mathrm{mol} / \mathrm{L})$ & 114.9 & 106.1 & 123.8 & $<0.0001$ \\
\hline BUN (mg/dL) & 8.6 & 8.2 & 9.6 & 0.0001 \\
\hline Sodium (mmol/L) & 139 & 139 & 139 & 0.038 \\
\hline $\mathrm{EF}(\%)$ & 25 & 30 & 25 & 0.052 \\
\hline Time from presentation to randomization (h) & 18.1 & 18.8 & 17.3 & 0.094 \\
\hline Ischaemic aetiology $(\%)$ & 60.6 & 61.1 & 60.2 & 0.77 \\
\hline On beta-blocker (\%) & 75.5 & 78.0 & 73.0 & 0.10 \\
\hline On ACE inhibitor or ARB (\%) & 64.2 & 65.4 & 63.1 & 0.51 \\
\hline On aldosterone antagonist (\%) & 24.6 & 27.5 & 21.8 & 0.060 \\
\hline
\end{tabular}

ACE, angiotensin-converting enzyme; ARB, angiotensin receptor blocker; BP, blood pressure; BUN, blood urea nitrogen; cTnl, cardiac troponin I; EF, ejection fraction; NT-proBNP, N-terminal pro brain natriuretic peptide. 
$P=0.15)$ or multivariable (OR $1.04,95 \% \mathrm{Cl} 0.92-1.18 ; P=0.51)$ analyses. For 180-day mortality, elevations of baseline cTnl were associated with higher mortality in univariable analysis (HR 1.25, 95\% Cl 1.13-1.38 per doubling of cTnl; $P<0.0001$ ), but were no longer significant after adjustment for other predictors (adjusted HR 1.11, 95\% Cl 0.99-1.25 per doubling of cTnl; $P=$ 0.086).

\section{Change in troponin status and outcomes}

A total of 685 patients had paired samples from both the baseline and 48-72 h time point available for analysis. Event rates in patients with paired samples were generally similar to those in patients without paired samples available (data not shown). In general, cTnl levels from baseline to the 48-72 h time point were highly correlated $(r=0.77 ; P<0.001)$. There was no significant association between the absolute change in troponin from baseline to $48-72 \mathrm{~h}$ and either 30-day mortality $(P=0.063)$ or the composite of 30-day death or heart failure hospitalization $(P=0.14)$. When troponin change was dichotomized at a $20 \%$ increase, 30-day event rates were higher for those with a troponin increase (Table 4), and a 20\% increase in troponin was a significant predictor of 30-day death $(P=0.012)$ but not 30-day death or HF hospitalization $(P=0.304)$. Finally, we assessed the clinical significance of a rise or fall in troponin that crossed the 99th percentile

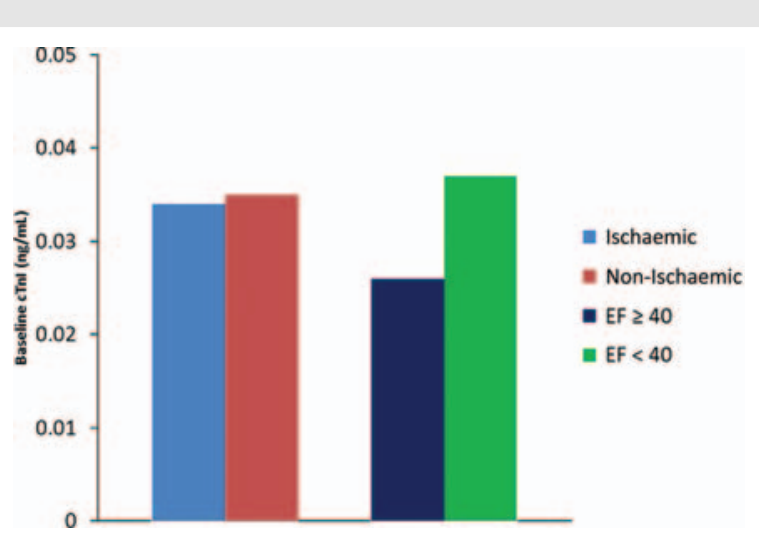

Figure 2 Relationship between heart failure aetiology, ejection fraction (EF), and baseline cardiac troponin I (cTnl).
URL (consistent with the universal definition for myocardial infarction). Event rates for 30-day endpoints (death and death or HF rehospitalization) based on changes in troponin status are shown in Figure 4. Although crude event rates were numerically highest for patients with persistently elevated cTnl, there was no clear and consistent relationship between change in troponin category and 30-day outcome for either mortality or the composite of mortality and rehospitalization. Notably, patients whose cTnl went from low to high during the course of treatment (presumably suggestive of ongoing or worsening myocardial injury) actually had the lowest event rate for the composite of death or HF hospitalization at 30 days.

When both baseline and 48-72 h log(cTnl) were entered together into a logistic regression model for 30-day mortality, the 48-72 h cTnl level remained a significant predictor $(P=0.04)$, whereas the baseline $\mathrm{cTn}$ l level did not $(P=0.81)$, suggesting that the most recent value provided the most prognostic information. In a similar analysis examining change from baseline to 30 - and 180-day outcomes, the 30-day cTnl level remained a significant predictor $(P=0.0001)$ whereas baseline $c T n l$ did not $(P=0.434)$.

\section{Interaction between troponin status and treatment}

Given that ASCEND-HF was a randomized controlled study of nesiritide, we evaluated the association between treatment assignment (nesiritide or placebo) and troponin status using interaction terms in the relevant multivariable model for each endpoint. There was no evidence of a differential effect of nesiritide treatment based on troponin status for either dyspnoea at $24 \mathrm{~h}(P=0.56$ for treatment $\times \mathrm{cTnl}$ interaction) or for the 30-day composite of death or HF rehospitalization $(P=0.42$ for treatment $\times \mathrm{cTnl}$ interaction). Additionally, we investigated whether the changes in cTnl during initial ADHF treatment differed by treatment assignment (nesiritide or placebo). There was no significant difference between $72 \mathrm{~h} \mathrm{cTnl}$ (while controlling for baseline troponin) based on treatment assignment $(P=0.56)$, suggesting that nesiritide treatment did not affect changes in $c T n l$ over time.

\section{Discussion}

The ongoing development of more sensitive assays for troponin has led to uncertainty about the clinical significance of detectable

Table 2 Predictors of higher baseline troponin I levels by linear regression

\begin{tabular}{|c|c|c|c|c|}
\hline Variable & Coefficient & $95 \% \mathrm{Cl}$ & F-value & $P$-value \\
\hline Log(NT-proBNP) & 0.212 & $0.140-0.284$ & 33.38 & $<0.0001$ \\
\hline Region $^{\mathrm{a}}$ & NA & & 5.21 & 0.0004 \\
\hline $\log (B \cup N)$ & 0.171 & $0.015-0.327$ & 4.66 & 0.0313 \\
\hline Potassium & 0.122 & $0.007-0.237$ & 4.30 & 0.0385 \\
\hline No diabetes & -0.150 & -0.294 to -0.022 & 4.13 & 0.0424 \\
\hline No atrial fibrillation & -0.141 & -0.286 to 0.004 & 3.62 & 0.0576 \\
\hline
\end{tabular}

BUN, blood urea nitrogen; $\mathrm{Cl}$, confidence interval; NT-proBNP, N-terminal pro brain natriuretic peptide.

${ }^{a}$ North America, Latin America, Central Europe, Western Europe, or Asia. 
Table 3 Baseline troponin I levels and outcomes

\begin{tabular}{|c|c|c|c|c|c|c|c|c|}
\hline \multirow[t]{2}{*}{ Endpoint } & \multicolumn{4}{|l|}{ Unadjusted } & \multicolumn{4}{|l|}{ Adjusted $^{\mathbf{a}}$} \\
\hline & ${ }^{\prime} R^{b}$ or $H R^{b}$ & $95 \% \mathrm{Cl}$ & $x^{2}$ & P-value & $\dddot{O R}^{\mathrm{b}}$ or $\mathrm{HR}^{\mathrm{b}}$ & $95 \% \mathrm{Cl}$ & $\dddot{2}$ & P-value \\
\hline Death at 30 days & 1.234 & $1.014-1.501$ & 4.42 & 0.035 & 1.106 & $0.887-1.379$ & 0.80 & 0.37 \\
\hline Death/rehospitalization at 30 days & 1.090 & $0.970-1.224$ & 2.08 & 0.15 & 1.043 & $0.919-1.184$ & 0.43 & 0.51 \\
\hline Death at 180 days & 1.246 & $1.126-1.377$ & 18.3 & $<0.0001$ & 1.108 & $0.985-1.247$ & 2.95 & 0.086 \\
\hline Improved dyspnoea at $6 \mathrm{~h}$ & 1.008 & $0.930-1.093$ & 0.04 & 0.84 & 1.037 & $0.933-1.153$ & 0.45 & 0.502 \\
\hline Improved dyspnoea at $24 \mathrm{~h}$ & 0.886 & $0.788-0.991$ & 4.02 & 0.045 & 0.845 & $0.724-0.988$ & 4.47 & 0.035 \\
\hline Death or worsening HF prior to discharge & 1.310 & $1.115-1.538$ & 10.8 & 0.001 & 1.336 & $1.101-1.620$ & 8.65 & 0.013 \\
\hline Length of stay ${ }^{c}$ & 0.931 & $0.894-0.970$ & 11.7 & 0.001 & 0.946 & $0.905-0.988$ & 6.14 & 0.013 \\
\hline \multicolumn{9}{|c|}{$\begin{array}{l}\mathrm{Cl} \text {, confidence interval; } \mathrm{HF} \text {, heart failure; HR, hazard ratio; OR, odds ratio. } \\
\text { aAdjusted models using th ecovariates listed in Appendix for each endpoint. } \\
\text { ber doubling of cardiac troponin I. }\end{array}$} \\
\hline
\end{tabular}

or elevated troponin levels in patients with HF. Specifically, improvements in analytical sensitivity have transformed circulating troponin from a biomarker that was only detectable in a minority of patients to one that is detectable in the vast majority of patients with HF and elevated (above the 99th percentile URL) in a substantial minority.

In the current analysis of a substudy from the ASCEND-HF trial, we found a high prevalence of baseline cTnl levels that were detectable $(78 \%)$ or elevated above the 99 th percentile URL (50\%) using a contemporary sensitive troponin assay. The prevalence of cTnl elevation in our study was generally higher than in previously published reports in ADHF, where the prevalence of troponin elevation above the 99th percentile URL has ranged from $6 \%$ to 83\%. ${ }^{4,5,10-13}$ Previous studies of troponin prevalence have been either small single-centre studies ${ }^{5,14-16}$ or pooled data from large registries such as ADHERE and EFFECT ${ }^{4,11}$ and have generally used older assays or pooled clinical data from multiple sites using a variety of assays. Thus, the current analysis from ASCEND-HF is the largest study to date reporting troponin data in ADHF patients using a centralized core laboratory and a contemporary sensitive assay. Additionally, our study utilized carefully adjudicated clinical events using a blinded endpoints committee, further adding to the validity of our findings.

The ongoing evolution of troponin assays has resulted in assays of markedly greater sensitivity (at least 10-fold); not surprisingly, this led to substantial increases in the proportion of HF patients with detectable (i.e. above the lower limit of detection) and elevated (i.e. above the 99th percentile URL) troponin. Consistent with this trend, a recent single-centre study by Xue et al. demonstrated, using an experimental ultrasensitive nanoparticle assay, that the median troponin value in an ADHF population was $29.5 \mathrm{ng} / \mathrm{L}$ (>4-fold greater than the 99th percentile URL for that assay) and all ADHF patients studied had detectable troponin. ${ }^{14}$ These progressive improvements in analytical sensitivity have resulted in the commonly used distinction of troponin as 'positive' or 'negative' becoming increasingly meaningless in the context of ADHF, where the vast majority of patients have detectable troponin using contemporary sensitive assays.
In our analysis, elevations in baseline cTnl were independent predictors of events during the acute index hospitalization (worsening or persistent HF or death as well as increased length of stay) and modestly predicted lack of dyspnoea improvement at $24 \mathrm{~h}$. In this analysis, cTnl levels, while predictive of in-hospital outcomes, were not independent predictors of post-discharge outcomes at 30 or 180 days after adjustment for other known predictors. These results are generally consistent with previous results from ADHERE examining in-hospital events, ${ }^{4}$ but are in contrast to previous published studies that have consistently shown troponin elevation to be an independent predictor of postdischarge outcomes in ADHF, $5,10,11,16-18$ The reasons underlying the difference between our results and previous data in ADHF are speculative. Our study is several fold larger than any previous study (excluding the two large registry studies), suggesting that limited statistical power (i.e. type II error) is an unlikely explanation for our results. Our study included relatively robust adjustment for other covariates, which may have served to attenuate the prognostic significance of troponin in the face of other strong predictors of outcome. The ASCEND-HF study excluded patients with troponin $>5$-fold the URL, and so the exclusion of patients with very elevated troponin levels may have limited our ability to detect associations between troponin and outcomes. Finally, publication bias may contribute to the lack of other reports in the literature showing no association between troponin and post-discharge outcomes.

Changes in troponin status during initial treatment for ADHF have been proposed as potentially important signals in drug development. ${ }^{19}$ Previous studies in both chronic and acute HF have suggested that changes in troponin over time are associated with greater risk. ${ }^{18,20}$ In the current analysis, troponin values were relatively stable over the initial $48-72 \mathrm{~h}$ of ADHF therapy. Substantial increases in troponin ( $>20 \%$ ) were associated with risk for 30-day mortality but not for the composite of death or HF rehospitalization. The most recent value available provided the most important prognostic information in predicting outcomes, consistent with recent data on the natriuretic peptides. ${ }^{21}$ Of particular interest in our analysis were patients whose troponin either rose above the 99th percentile URL $(5 \% ; n=33)$ or fell below the 99th 

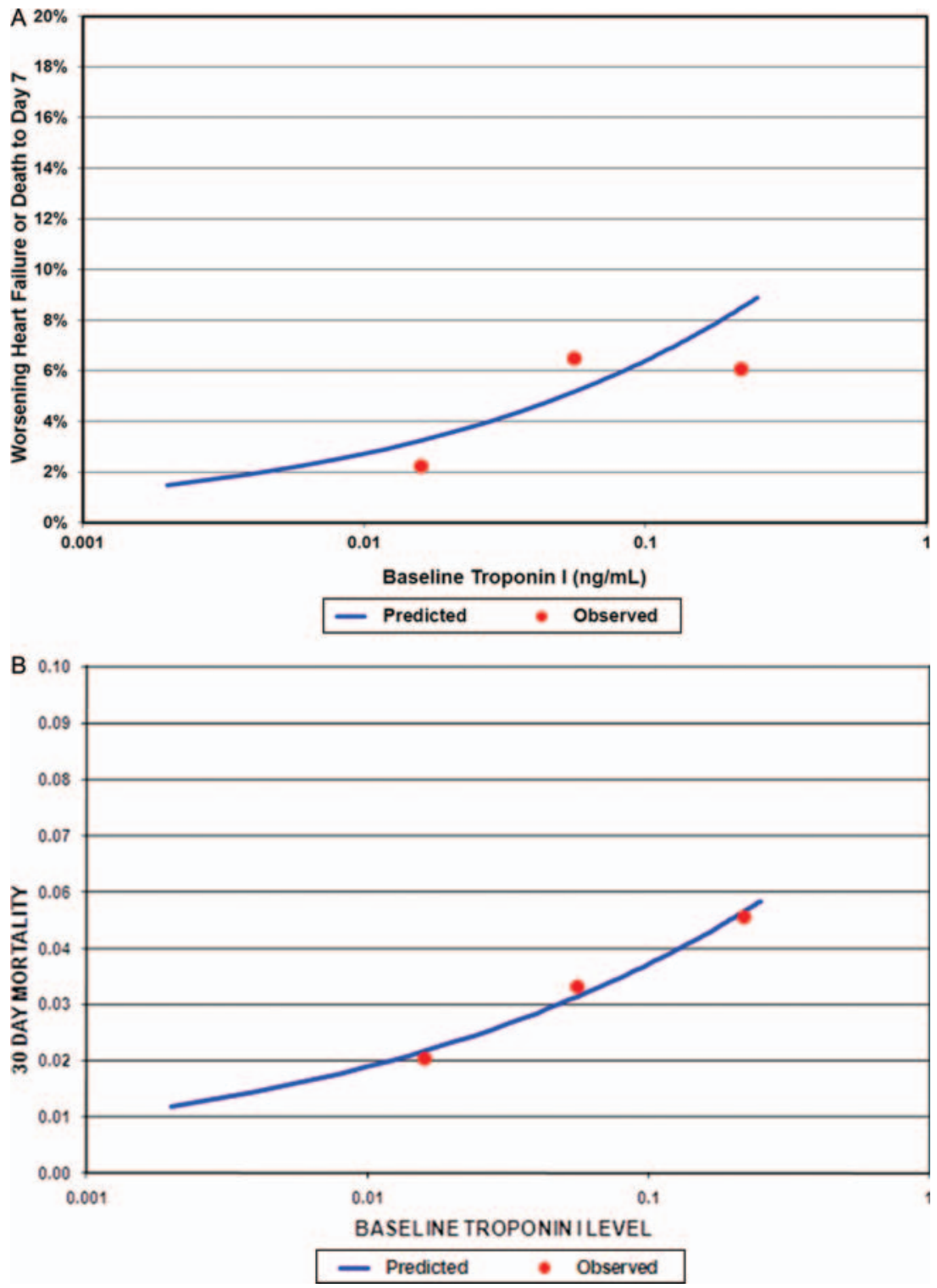

Figure 3 Continuous relationship between baseline troponin I and $(A)$ in-hospital worsening heart failure or death to day 7, and (B) 30-day mortality.

Table 4 Change in troponin from baseline to $48-72 \mathrm{~h}$ and 30-day outcomes

\begin{tabular}{|c|c|c|c|c|}
\hline \multirow[t]{2}{*}{ Endpoint, \% (n/N) } & \multicolumn{4}{|c|}{ Event rates based on $\mathbf{2 0} \%$ increase in troponin } \\
\hline & No $20 \%$ increase & $20 \%$ increase & P-value & \\
\hline 30-day death & $2.3 \%(10 / 441)$ & $7.7 \%(7 / 91)$ & 0.012 & \\
\hline 30-day death/HF rehospitalization & $12.5 \%(55 / 441)$ & $16.5 \%(15 / 91)$ & 0.304 & \\
\hline \multirow[t]{2}{*}{ Endpoint } & \multicolumn{4}{|c|}{ Absolute change in troponin and 30-day endpoints } \\
\hline & OR & $95 \% \mathrm{Cl}$ & Wald $\chi^{2}$ & P-value \\
\hline 30-day death & 0.762 & $0.572-1.015$ & 3.46 & 0.063 \\
\hline 30-day death/HF rehospitalization & 0.856 & $0.696-1.054$ & 2.14 & 0.144 \\
\hline
\end{tabular}

$\mathrm{Cl}$, confidence interval; $\mathrm{HF}$, heart failure; OR, odds ratio. 


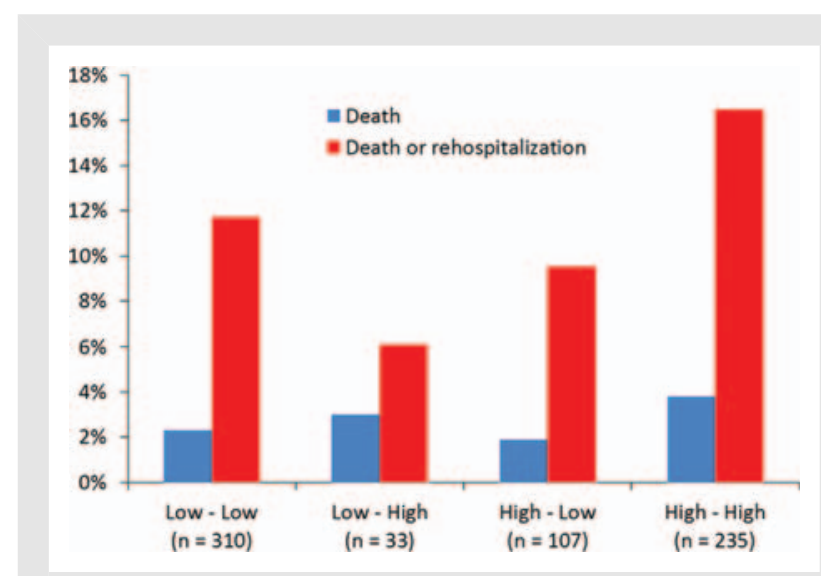

Figure 4 Thirty-day event rates by change in troponin category from baseline to $48-72 \mathrm{~h}$. High and low troponin categories based on level above or below the 99th percentile upper reference limit.

percentile URL $(16 \% ; n=107)$ between baseline and $48-72 \mathrm{~h}$ measurements. Given that the clinical presentation of acute HF and ACS substantially overlaps, this subgroup of patients satisfies many of the diagnostic criteria for acute myocardial infarction using the current universal definition, which requires a rise and/ or fall in cardiac troponin with at least one value above the 99th percentile URL in the setting of appropriate clinical symptoms. ${ }^{9}$ Absolute changes in troponin in these groups were modest, with a median rise of $0.031 \mathrm{ng} / \mathrm{mL}$ in the low-high group and a median fall of $0.025 \mathrm{ng} / \mathrm{mL}$ in the high-low group. Despite these changes in troponin status during acute therapy, there was no clear or consistent pattern in event rates based on these categories, as shown in Figure 4. These data call into question the clinical significance of modest changes in troponin in the context of acute $\mathrm{HF}$, a finding that may have potential implications for the use of troponin monitoring to identify cardiotoxicity in drug development. ${ }^{22}$ Larger increases in troponin during acute treatment may have prognostic implications for post-discharge events.

Given the importance of the 99th percentile URL in current guidelines for diagnosing myocardial infarction, ${ }^{9}$ we evaluated whether there was any evidence for a threshold effect (a change in the slope of the risk curve) at this level of cTnl values. Graphical evaluation of the continuous relationship between cTnl and risk of in-hospital worsening HF/death to day 7 (Figure 3A) or 30-day mortality (Figure $3 B$ ) did not suggest a threshold effect at any particular level of cTnl. Combined with our data showing little significance of the changes in troponin categories based on troponin levels above or below this threshold, the data from ASCEND-HF generally raise questions regarding the relevance of the 99th percentile URL in the evaluation and management of patients with ADHF.

Important limitations of our study include the selection bias inherent in clinical trial populations, as well as the fact that patients with clinical evidence of ACS and elevations of troponin $>5 \times$ the URL were excluded from the ASCEND-HF trial. If anything, these exclusion criteria would bias our data towards an underestimate of the prevalence of elevated troponin levels in less selected ADHF populations. However, this limitation may also have led us to underestimate the prognostic importance of troponin elevations in ADHF patients with a broader range of troponin values. The majority of our substudy population was from North America, potentially limiting the generalizability of our results. Patients in ASCEND-HF could be randomized as long as $24 \mathrm{~h}$ after initiation of intravenous therapy, and thus baseline cTnl levels in our study may have differed from those that would have been obtained at initial presentation. The relatively long time window between baseline and follow-up sampling (48-72 h) may have obscured shorter term changes in troponin levels. Although our study was relatively large compared with other similar studies in the literature, the total number of events was still small, which may have limited our statistical power. Finally, our study was a retrospective analysis of data from a clinical trial, and thus cannot establish cause and effect.

The ongoing development of troponin assays of progressively greater sensitivity necessitates a change in thinking about troponin as a dichotomous variable (with associated 'cut-off points') to a continuous prognostic variable (similar to the natriuretic peptides), a change that has resulted in substantial uncertainty in the clinical community. ${ }^{23}$ In this analysis of a large clinical trial population, cTnl levels were associated with in-hospital outcomes but not postdischarge outcomes in a large cohort of patients with ADHF. Given that these results differ from those of other recent analyses, ${ }^{24,25}$ they will require confirmation in other data sets. Further research with contemporary assays is needed to define the appropriate role of troponin as a biomarker in the clinical care of patients with HF.

\section{Funding}

Johnson \& Johnson.

Conflict of interest: G.M.F.: research grants from Johnson \& Johnson, Roche Diagnostics, Critical Diagnostics, and BG Medicine. A.F.H., K.D., J.J.,V.M., R.M.C., and C.M.O.: research grants from Johnson \& Johnson. P.W.A.: research grants from Johnson \& Johnson and Ortho Biotech. G.C.F. : consultant/advisory board for Scios and Novartis. A.A.V., J.B., and B.M.M.: consultant/advisory board for Johnson \& Johnson. M.M.: consultant/advisory board for Corthera, Daiichi, Novartis, and Serrvier. R.W.T: consultant/advisory board for St. Jude Medical. S.D.A.: honoraria and consultant/advisory board for Alere Inc. (Europe), Thermo Fisher Scientific, and Vifor Pharma. R.C.S.: research support and consultant/advisory board for Johnson \& Johnson. All other authors have no conflicts to declare.

\section{Appendix}

Final covariates for multivariable adjustment for each endpoint were based on multivariable modelling from the overall ASCEND-HF cohort, and then applied to models for each endpoint in the troponin subset. Final adjustment covariates for each endpoint are shown below.

\section{Death at 30 days}

Age, $\log (B U N)$, serum sodium, hypotension (baseline), dyspnoea at rest (baseline). 


\section{Death or HF rehospitalization at $\mathbf{3 0}$ days}

Hypotension (baseline), serum sodium, age, $\log (\mathrm{BUN})$, history of cerebrovascular disease (baseline), log(creatinine), history of depression, dyspnoea at rest (baseline), elevated jugular venous pressure (JVP), history of chronic respiratory disease.

\section{Length of stay}

Hypotension (baseline), serum sodium, age, $\log (\mathrm{BUN})$, history of cerebrovascular disease (baseline), log(creatinine), history of depression, dyspnoea at rest (baseline), elevated JVP, history of chronic respiratory disease.

\section{Dyspnoea at $6 \mathrm{~h}$}

Age, serum sodium, respiratory rate (baseline), congestion on chest X-ray, race, hypotension (baseline), haemoglobin, $\log (\mathrm{BUN})$, New York Heart Association (NYHA) class prior to decompensation.

\section{Dyspnoea at $24 \mathrm{~h}$}

Age, serum sodium, respiratory rate (baseline), congestion on chest X-ray, race, hypotension (baseline), haemoglobin, NYHA class prior to decompensation.

\section{Death at 180 days}

Age, $\log (\mathrm{BUN})$, serum sodium, hypotension (baseline), dyspnoea at rest (baseline).

\section{References}

1. Kociol RD, Pang PS, Gheorghiade M, Fonarow GC, O'Connor CM, Felker GM. Troponin elevation in heart failure prevalence, mechanisms, and clinical implications. J Am Coll Cardiol 2010;56:1071-1078.

2. Freda BJ, Tang WH, Van Lente F, Peacock WF, Francis GS. Cardiac troponins in renal insufficiency: review and clinical implications. J Am Coll Cardiol 2002;40: 2065-2071.

3. Biolo A, Fisch M, Balog J, Chao T, Schulz PC, Ooi H, Siwik D, Colucci WS. Episodes of acute heart failure syndrome are associated with increased levels of troponin and extracellular matrix markers. Circ Heart Fail 2010;3:44-50.

4. Peacock WF IV, De Marco T, Fonarow GC, Diercks D, Wynne J, Apple FS, $\mathrm{Wu} \mathrm{AH}$. Cardiac troponin and outcome in acute heart failure. N Engl J Med 2008;358:2117-2126.

5. Metra M, Nodari S, Parrinello G, Specchia C, Brentana L, Rocca P, Fracassi F, Bordonali T, Milani P, Danesi R, Verzura G, Chiari E, Dei Cas L. The role of plasma biomarkers in acute heart failure. Serial changes and independent prognostic value of NT-proBNP and cardiac troponin-T. Eur J Heart Fail 2007;9: $776-786$.

6. O'Connor CM, Starling RC, Hernandez AF, Armstrong PW, Dickstein K, Hasselblad V, Heizer GM, Komajda M, Massie BM, McMurray JJ, Nieminen MS, Reist CJ, Rouleau JL, Swedberg K, Adams KF Jr, Anker SD, Atar D, Battler A, Botero R, Bohidar NR, Butler J, Clausell N, Corbalán R, Costanzo MR, Dahlstrom U, Deckelbaum LI, Diaz R, Dunlap ME, Ezekowitz JA, Feldman D, Felker GM, Fonarow GC, Gennevois D, Gottlieb SS, Hill JA, Hollander JE, Howlett JG, Hudson MP, Kociol RD, Krum H, Laucevicius A, Levy WC, Méndez GF, Metra M, Mittal S, Oh BH, Pereira NL, Ponikowski P, Tang WH, Tanomsup S, Teerlink JR, Triposkiadis F, Troughton RW, Voors AA, Whellan DJ, Zannad F, Califf RM. Effect of nesiritide in patients with acute decompensated heart failure. N Engl J Med 2011;365:32-43.

7. Hernandez AF, O'Connor CM, Starling RC, Reist C], Armstrong PW, Dickstein K, Lorenz TJ, Gibler WB, Hasselblad V, Komajda M, Massie B, McMurray JJ, Nieminen M, Rouleau JL, Swedberg K, Califf RM. Rationale and design of the Acute Study of Clinical Effectiveness of Nesiritide in Decompensated Heart Failure Trial (ASCEND-HF). Am Heart J 2009;157:271-277.

8. Apple FS. A new season for cardiac troponin assays: it's time to keep a scorecard. Clin Chem 2009;55:1303-1306.
9. Thygesen K, Alpert JS, White HD. Universal definition of myocardial infarction. Circulation 2007;116:2634-2653.

10. Del Carlo CH, Pereira-Barretto AC, Cassaro-Strunz C, Latorre Mdo R, Ramires JA. Serial measure of cardiac troponin $T$ levels for prediction of clinical events in decompensated heart failure. J Card Fail 2004;10:43-48.

11. You JJ, Austin PC, Alter DA, Ko DT, Tu JV. Relation between cardiac troponin I and mortality in acute decompensated heart failure. Am Heart J 2007;153 462-470.

12. Gheorghiade M, Gattis Stough W, Adams KF Jr, Jaffe AS, Hasselblad V, O'Connor CM. The Pilot Randomized Study of Nesiritide Versus Dobutamine in Heart Failure (PRESERVD-HF). Am J Cardiol 2005;96:p18G-25G.

13. Kuwabara $Y$, Sato $Y$, Miyamoto $T$, Taniguchi R, Matsuoka T, Isoda K, Yamane K, Nishi K, Fujiwara H, Takatsu Y. Persistently increased serum concentrations of cardiac troponin in patients with acutely decompensated heart failure are predictive of adverse outcomes. Circ J 2007;71:1047-1051.

14. Xue Y, Clopton P, Peacock WF, Maisel AS. Serial changes in high-sensitive troponin I predict outcome in patients with decompensated heart failure. Eur J Heart Fail 2011;13:37-42.

15. Perna ER, Macin SM, Cimbaro Canella JP, Szyszko A, Franciosi V, Vargas Morales W, Bayol AP, Kriskovich JO, Medina F, Gonzalez Arjol B, Brizuela M. Importance of early combined $\mathrm{N}$-terminal pro-brain natriuretic peptide and cardiac troponin $\mathrm{T}$ measurements for long-term risk stratification of patients with decompensated heart failure. J Heart Lung Transplant 2006;25:1230-1240.

16. Pascual-Figal DA, Manzano-Fernandez S, Boronat M, Casas T, Garrido IP, Bonaque JC, Pastor-Perez F, Valdés M, Januzzi JL. Soluble ST2, high-sensitivity troponin $\mathrm{T}$ - and $\mathrm{N}$-terminal pro-B-type natriuretic peptide: complementary role for risk stratification in acutely decompensated heart failure. Eur J Heart Fail 2011;13:718-725.

17. Parenti N, Bartolacci S, Carle F, Angelo F. Cardiac troponin I as prognostic marker in heart failure patients discharged from emergency department. Intern Emerg Med 2008;3:43-47.

18. O'Connor CM, Fiuzat M, Lombardi C, Fujita K, Jia G, Davison BA, Cleland J, Bloomfield D, Dittrich HC, Delucca P, Givertz MM, Mansoor G, Ponikowski P, Teerlink JR, Voors AA, Massie BM, Cotter G, Metra M. Impact of serial troponin release on outcomes in patients with acute heart failure: analysis from the PROTECT pilot study. Circ Heart Fail 2011;4:724-732.

19. Felker GM, Pang PS, Adams KF, Cleland JG, Cotter G, Dickstein K, Filippatos GS, Fonarow GC, Greenberg BH, Hernandez AF, Khan S, Komajda M, Konstam MA, Liu PP, Maggioni AP, Massie BM, McMurray JJ, Mehra M, Metra M, O'Connell J, O'Connor CM, Pina IL, Ponikowski P, Sabbah HN, Teerlink JR, Udelson JE, Yancy CW, Zannad F, Gheorghiade M. Clinical trials of pharmacological therapies in acute heart failure syndromes: lessons learned and directions forward. Circ Heart Fail 2010;3:314-325.

20. Miller WL, Hartman KA, Burritt MF, Grill DE, Jaffe AS. Profiles of serial changes in cardiac troponin $\mathrm{T}$ concentrations and outcome in ambulatory patients with chronic heart failure. J Am Coll Cardiol 2009;54:1715-1721.

21. Kociol RD, Horton JR, Fonarow GC, Reyes EM, Shaw LK, O'Connor CM, Felker GM, Hernandez AF. Admission, discharge, or change in B-type natriuretic peptide and long-term outcomes: data from Organized Program to Initiate Lifesaving Treatment in Hospitalized Patients with Heart Failure (OPTIMIZE-HF) linked to Medicare claims. Circ Heart Fail 2011;4:628-636.

22. Newby LK, Rodriguez I, Finkle J, Becker RC, Hicks KA, Hausner E, Chesler R, Harper C, Targum S, Berridge BR, Lewis E, Walker DB, Dollery C, Turner JR, Krucoff MW. Troponin measurements during drug development - considerations for monitoring and management of potential cardiotoxicity: an educational collaboration among the Cardiac Safety Research Consortium, the Duke Clinical Research Institute, and the US Food and Drug Administration. Am Heart J 2011; 162:64-73.

23. Jesse RL. On the relative value of an assay versus that of a test: a history of troponin for the diagnosis of myocardial infarction. I Am Coll Cardiol 2010;55: 2125-2128.

24. Metra M, Bettari L, Pagani F, Lazzarini V, Lombardi C, Carubelli V, Bonetti G, Bugatti S, Parrinello G, Caimi L, Felker G, Cas Dei L.. Troponin T levels in patients with acute heart failure: clinical and prognostic significance of their detection and release during hospitalisation. Clin Res Cardiol 2012;101:1-10.

25. Arenja N, Reichlin T, Drexler B, Reichlin T, Drexler B, Oshima S, Denhaerynck K, Haaf P, Potocki M, Breidthardt T, Noveanu M, Stelzig C, Heinisch C, Twerenbold R, Reiter M, Socrates T, Mueller C. Sensitive cardiac troponin in the diagnosis and risk stratification of acute heart failure. J Intern Med 2012;271: 598-607. 